\title{
Quantum Entanglement from Classical Trajectories
}

\author{
Johan E. Runeson $\odot^{*}$ and Jeremy O. Richardson $\oplus^{\dagger}$ \\ Laboratory of Physical Chemistry, ETH Zürich, 8093 Zürich, Switzerland
}

(Received 3 May 2021; revised 20 August 2021; accepted 5 November 2021; published 15 December 2021)

\begin{abstract}
A long-standing challenge in mixed quantum-classical trajectory simulations is the treatment of entanglement between the classical and quantal degrees of freedom. We present a novel approach that describes the emergence of entangled states entirely in terms of independent and deterministic Ehrenfestlike classical trajectories. For a two-level quantum system in a classical environment, this is derived by mapping the quantum system onto a path-integral representation of a spin $\frac{1}{2}$. We demonstrate that the method correctly accounts for coherence and decoherence and thus reproduces the splitting of a wave packet in a nonadiabatic scattering problem. This discovery opens up a new class of simulations as an alternative to stochastic surface-hopping, coupled-trajectory, or semiclassical approaches.
\end{abstract}

DOI: 10.1103/PhysRevLett.127.250403

Introduction.-Many important phenomena across physics and chemistry are best described by a small quantum system and a large classical environment; for example, light-matter interaction, chemical reactions, and qubits. A prototypical model for all these examples is the crossing between two electronic states coupled to classical nuclei. As it is intractable to treat the entire problem with quantum mechanics, it is necessary to simulate the coupled quantumclassical dynamics directly [1]. Deriving an approach which is both computationally efficient and accurate is, however, a highly nontrivial task. The simplest method based on classical trajectories is Ehrenfest dynamics, also known as mean-field theory (MFT) [2]. While this approach is computationally efficient, it completely neglects quantum entanglement between the system and the environment. As a consequence, it is unable to describe the branching of a nuclear wave packet in a nonadiabatic scattering problem [3].

Over the last decades, a considerable effort has been invested in the development of more accurate trajectorybased methods. A popular approach, especially in simulations of photochemistry, is Tully's fewest switches surface hopping (FSSH) [4-6], whose trajectories take stochastic jumps to simulate wave-packet branching. Although its original form is known to suffer from overcoherence, there have been many suggestions to introduce decoherence corrections [7,8] with little consensus that there is a definitive solution. Another known way to include entanglement is to use coupled trajectories, either on top of

Published by the American Physical Society under the terms of the Creative Commons Attribution 4.0 International license. Further distribution of this work must maintain attribution to the author(s) and the published article's title, journal citation, and DOI.
Ehrenfest [9] or surface hopping [10] or through methodologies such as the exact-factorization framework [11-13], $a b$ initio multiple spawning [14], the quantum-classical Liouville equation [15], or Bohmian dynamics [16]. A third possibility is to use interference between path histories and weight Ehrenfest-like trajectories (obtained from a mapping scheme [17-19] that has a close relation to the Stratonovich-Weyl representation used in the present Letter $[20,21]$ ) by phases and prefactors derived from a semiclassical propagator based on a real-time path integral [22-25]. At first sight, decoherence and entanglement appear to be inherently quantum phenomena which cannot be described with a fully classical simulation [26]. However, in this Letter, we introduce a new approach that, in contrast to the three approaches described above, can capture these effects based on independent and deterministic classical trajectories.

Our theory is based on the Stratonovich-Weyl (SW) phase-space representation of the quantum system, which is a Wigner representation of discrete spaces [27,28]. For simplicity, we consider only the two-level case, which employs the well-known isomorphism to a spin $S=\frac{1}{2}$ system, and represent the spin by a classical vector of length $\sqrt{S(S+1)}$. We propose to extend this approach to a path integral of spin vectors, where the centroid of the spin path determines the dynamics, and the initial configuration specifies the weight of each trajectory. This weight, which is preserved along the trajectory, contains the information necessary to recover the quantum entanglement between the system and the environment.

Method.-First, consider an isolated two-level quantum system with density matrix $\hat{\rho}$. A convenient classical analog for this system is given by the Stratonovich-Weyl W representation [29], which expresses the expectation value of an operator $\hat{A}$ by the integral 


$$
\operatorname{tr}[\hat{\rho} \hat{A}]=\int d^{2} \boldsymbol{s} \rho(\boldsymbol{s}) A(\boldsymbol{s}) .
$$

The classical functions are defined as

$$
\rho(\boldsymbol{s})=\operatorname{tr}[\hat{\rho} \hat{w}(\boldsymbol{s})], \quad \hat{w}(\boldsymbol{s})=\frac{1}{2} \hat{\mathcal{I}}+\boldsymbol{s} \cdot \hat{\boldsymbol{\sigma}},
$$

and likewise for $A(\boldsymbol{s})$, where $\hat{w}(\boldsymbol{s})$ is the SW kernel, $\hat{\mathcal{I}}$ is the $2 \times 2$ identity matrix, $\hat{\sigma}=\left[\hat{\sigma}_{x}, \hat{\sigma}_{y}, \hat{\sigma}_{z}\right]$ are the Pauli matrices, and $s$ is a vector with magnitude $|s|=\sqrt{3} / 2$. For the integration measure we use the shorthand notation $\int d^{2} \boldsymbol{s}=(1 / 2 \pi) \int d \varphi d \theta \sin \theta$, where $\varphi$ and $\theta$ are spherical coordinates for the orientation of $\boldsymbol{s}$. Since each Cartesian component $s_{j}$ is the SW representation of the spin operator $\hat{S}_{j}=\frac{1}{2} \hat{\sigma}_{j}$, one can think of $s$ as a classical spin vector with the familiar quantum magnitude $\sqrt{S(S+1)}$ of a spin $S=\frac{1}{2}$ (where $\hbar=1$ throughout).

Next, consider the time evolution of the density matrix. As is well known, the dynamics of a two-level system is equivalent to that of a spin $\frac{1}{2}$ in an effective magnetic field $\boldsymbol{H}$, where the Hamiltonian is $\hat{H}=H_{0} \hat{\mathcal{I}}+\boldsymbol{H} \cdot \hat{\boldsymbol{S}}$. Using this decomposition, it is straightforward to write $H(s)=H_{0}+$ $\boldsymbol{H} \cdot \boldsymbol{s}$ and likewise $\rho(\boldsymbol{s})=\rho_{0}+\boldsymbol{\rho} \cdot \boldsymbol{s}$, where $\rho_{0}=\frac{1}{2}$ is fixed by the normalization. When the Liouville-von Neumann equation $d \hat{\rho} / d t=\mathrm{i}[\hat{\rho}, \hat{H}]$ is converted to its phase-space equivalent,

$$
\frac{d}{d t} \rho(\boldsymbol{s})=\mathrm{i} \operatorname{tr}[(\hat{\rho} \hat{H}-\hat{H} \hat{\rho}) \hat{w}(\boldsymbol{s})]=\boldsymbol{\rho} \cdot(\boldsymbol{s} \times \boldsymbol{H}),
$$

it follows that the standard precession formula for the classical spin vector $\dot{\boldsymbol{s}}=\boldsymbol{s} \times \boldsymbol{H}$ generates the correct quantum dynamics.

When coupled to a general classical environment (described by coordinates $x$, conjugate momenta $p$, and masses $m$ ), the total Hamiltonian

$$
\hat{H}=\left(\frac{p^{2}}{2 m}+U(x)\right) \hat{\mathcal{I}}+\left(\begin{array}{ll}
V_{1}(x) & \Delta^{*}(x) \\
\Delta(x) & V_{2}(x)
\end{array}\right)
$$

corresponds to $H_{0}(x, p)=p^{2} / 2 m+U(x)+\frac{1}{2}\left[V_{1}(x)+\right.$ $\left.V_{2}(x)\right]$ and $\boldsymbol{H}(x)=\left[2 \operatorname{Re} \Delta(x), 2 \operatorname{Im} \Delta(x), V_{1}(x)-V_{2}(x)\right]$. The corresponding equations of motion are [20]

$$
\dot{x}=\frac{p}{m}, \quad \dot{p}=-\frac{\partial H_{0}}{\partial x}-\frac{\partial \boldsymbol{H}}{\partial x} \cdot s,
$$

in addition to the spin dynamics as before. While these equations of motion are equivalent to those of Ehrenfest dynamics [30], the SW treatment differs in the initial distribution: while standard Ehrenfest starts from a unique vector $s$ of length $\frac{1}{2}$ (as in the Bloch-sphere picture), the SW approach averages over all initial spin directions in Eq. (1) and uses the magnitude $\sqrt{3} / 2$. We have recently found that the latter, called the linearized spin-mapping method, leads to a better prediction of population dynamics [20,21,31]. Other mapping approaches have also found an effective spin magnitude of $\sqrt{3} / 2$ to be optimal [32,33], and averaging over initial directions to be beneficial [34], even with the Ehrenfest spin length [35]. However, one important drawback is present in both Ehrenfest and linearized spin mapping; namely, that the dynamical quantization is lost. This has the unfortunate consequence that after a scattering event, the trajectories evolve on a weighted average of the two product potential energy surfaces, in contrast with the correct entangled state which splits into parts on one or the other surface [3]. We will now show that such quantization can be systematically reintroduced by representing the system by a path integral of spins. In contrast to standard spin coherent-state path integrals, we do not require paths to be continuous in the $N \rightarrow \infty$ limit and therefore do not have to deal with the difficulties that arise when restricting to such paths [36-38].

By construction, the SW representation has an inversion formula [29]

$$
\hat{\rho}=\int d^{2} \boldsymbol{s} \rho(\boldsymbol{s}) \hat{w}(\boldsymbol{s}),
$$

with the particular example of the identity, $\hat{\mathcal{I}}=\int d^{2} \boldsymbol{s} \hat{w}(\boldsymbol{s})$. By applying Eq. (6) to both operators in $\operatorname{tr}[\hat{\rho} \hat{A}]$ and inserting resolutions of the identity, we can generalize Eq. (1) to a path integral of $N$ spins,

$$
\begin{aligned}
\operatorname{tr}[\hat{\rho} \hat{A}]= & \int\left(\prod_{k=1}^{N} d^{2} \boldsymbol{s}_{k}\right) \operatorname{tr}\left[\prod_{k=1}^{N} \hat{w}\left(\boldsymbol{s}_{k}\right)\right] \\
& \times \frac{1}{N} \sum_{l=1}^{N} \rho\left(\boldsymbol{s}_{l}\right) \frac{1}{N} \sum_{m=1}^{N} A\left(\boldsymbol{s}_{m}\right),
\end{aligned}
$$

where we symmetrized over the indices $l$ and $m$ and used Eq. (1) for terms with $l=m$. Since $\rho(s)$ is linear in $s$ [Eq. (2)], it follows that $(1 / N) \sum_{l} \rho\left(\boldsymbol{s}_{l}\right)=\rho(\overline{\boldsymbol{s}})$ (and similar for $A)$, where we introduced the centroid $\bar{s}=(1 / N) \sum_{l} s_{l}$. The expression looks like a classical phase-space average with a weight function $g_{N}\left(\left\{\boldsymbol{s}_{k}\right\}\right) \equiv \operatorname{tr}\left[\prod_{k} \hat{w}\left(\boldsymbol{s}_{k}\right)\right]$. Note that if we had used $|\boldsymbol{s}|=\frac{1}{2}$, the weight function would reduce to that of standard spin coherent-state path integrals [39]. There are, however, significant advantages with our choice $|\boldsymbol{s}|=\sqrt{3} / 2$, as has been discussed in previous studies $[20,21]$.

A practical consideration is that $g_{N}\left(\left\{\boldsymbol{s}_{k}\right\}\right)$ is a complicated complex-valued function that varies rapidly for high $N$. However, since the observables depend only on $\bar{s}$ and not on the relative geometry, it is possible to rigorously integrate all degrees of freedom other than the centroid. This situation is analogous to the standard path-integral 


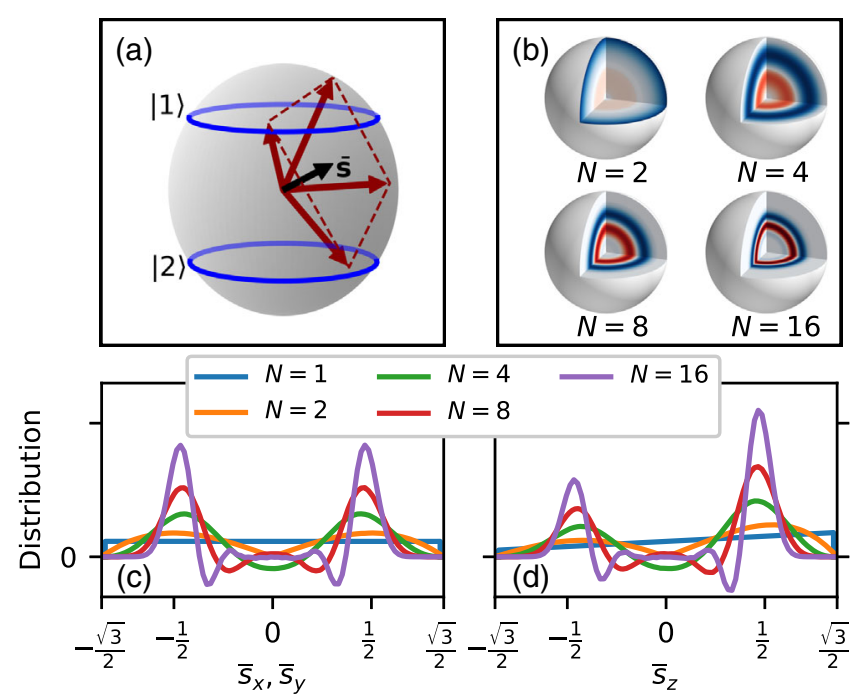

FIG. 1. (a) Sketch of a path of spin vectors (red arrows on the sphere) and their centroid (black arrow inside the sphere). The spin orientations corresponding to a pure state of the underlying two-level system are shown as blue circles at $s_{z}= \pm \frac{1}{2}$. (b) Weight of $\bar{s}$ [Eq. (8)], with positive contributions in blue and negative in red. The negative regions grow in importance for increasing number of spins, $N$. (c)-(d) Distribution of the $x, y$, and $z$ components of the spin centroid. The distributions become peaked around the quantum-mechanical eigenvalues $\pm \frac{1}{2}$ with increasing $N$, and the relative peak heights approach the corresponding expectation values of the density matrix, here plotted for $\hat{\rho}=\frac{2}{3}|1\rangle\left\langle 1\left|+\frac{1}{3}\right| 2\right\rangle\langle 2|$.

treatment of a harmonic oscillator (the reason being that the equations of motion are linear in both cases). Explicitly, we define

$$
G_{N}(\overline{\boldsymbol{s}}) \equiv \int\left(\prod_{k=1}^{N} d^{2} \boldsymbol{s}_{k}^{\prime}\right) g_{N}\left(\left\{\boldsymbol{s}_{k}^{\prime}\right\}\right) \delta^{(3)}\left(\overline{\boldsymbol{s}}-\overline{\boldsymbol{s}}^{\prime}\right) .
$$

Note that the weight function $g_{N}\left(\left\{\boldsymbol{s}_{k}\right\}\right)$ is invariant under global rotations of the spin vectors [40], so that $G_{N}$ is spherically symmetric, $G_{N}(\overline{\boldsymbol{s}})=G_{N}(\bar{s})$, where $\bar{s}=|\bar{s}|$. Equation (7) thus simplifies to

$$
\operatorname{tr}[\hat{\rho} \hat{A}]=\int d^{3} \overline{\boldsymbol{s}} G_{N}(\bar{s}) \rho(\overline{\boldsymbol{s}}) A(\overline{\boldsymbol{s}}) .
$$

Since the centroid of $N>1$ points on a sphere can reach any point inside the sphere as shown in Fig. 1(a), the integration domain of $\bar{s}$ is the ball $|\bar{s}| \leq \sqrt{3} / 2$. For $N=1$, we define $G_{1}(\bar{s})=(2 / 3 \pi) \delta(\bar{s}-\sqrt{3} / 2)$, which recovers Eq. (1).

The resulting universal function $G_{N}(\bar{s})$ has several important properties: (i) it depends only on the centroid magnitude and not on its direction, (ii) it is real valued, (iii) it is independent of the Hamiltonian and the initial conditions. In other words, even though its computation becomes exponentially hard with increasing $N$, it only has to be computed once for a given $N$, hence the name "universal." We have evaluated $G_{N}(\bar{s})$ numerically up to $N=16$ using Monte Carlo simulations [41]. Specialized algorithms will need to be developed to go beyond this. Figure 1(b) shows that the universal function consists of a few positive and negative domains, but the number of nodes seems to remain small for high $N$. The simulation will thus include trajectories with both positive and negative weights but this does not lead to a severe sign problem [41].

Next, we consider the distribution of the spin components, $\hat{S}_{j}$. Quantum mechanically these are expected to be quantized with the eigenvalues $\pm \frac{1}{2}$, but the integrand in Eq. (1) is smeared over all spin directions. However, as $N$ increases, the centroid distribution of Eq. (9) becomes peaked around $\bar{s}_{j}= \pm \frac{1}{2}$ for all $j \in\{x, y, z\}$, with heights that are consistent with the components of $\hat{\rho}$, as shown in Figs. 1(c)-1(d). In other words, the path-integral weight function $G_{N}(\bar{s})$ reintroduces the quantization to the system that is necessary for quantum entanglement.

Finally, consider time-dependent expectation values. From a similar argument as used in Eq. (3), it follows that a homogeneous precession of all spins, $\dot{\boldsymbol{s}}_{k}=\boldsymbol{s}_{k} \times \boldsymbol{H}$, ensures that Eq. (7) is valid also for the time-evolved density matrix. Consequently, the centroid evolves in the same way, $\dot{\overline{\boldsymbol{s}}}=\overline{\boldsymbol{s}} \times \boldsymbol{H}$, and hence, we do not need to keep track of the individual spin vectors. Since $G_{N}(\bar{s})$ is invariant under global rotations, its value is preserved by the dynamics, which has the important implication that Eq. (9) is valid for all times.

In this way, one can obtain the time-dependent expectation values of an isolated two-level system exactly using the centroid of the spin path integral with any value of $N$. For the coupled quantum-classical problem, we propose (in analogy to standard mixed quantum-classical methods) the approximation

$\operatorname{tr}[\hat{\rho} \hat{A}(t)] \approx \int d x d p d^{3} \overline{\boldsymbol{s}} G_{N}(\bar{s}) \rho(x, p, \overline{\boldsymbol{s}}) A\left(x_{t}, p_{t}, \overline{\boldsymbol{s}}_{t}\right)$,

where the phase-space version of the density operator $\rho(x, p, \bar{s})$ involves a Wigner transform of the environment in addition to the SW transform of the quantum system (and likewise for $A$ ). This equation is the main result in this Letter and will be referred to as the spin path-integral method. It is exact at $t=0$ and in the limit of an isolated system for all $N$. The $N=1$ case uses the same dynamics and spin distribution as the linearized spin-mapping method, which has been found to work well in many cases $[20,21]$. Next, we demonstrate that increasing $N$ further improves the results due to the quantization of the spin vector.

Results.-We have applied the spin path-integral method to Tully's seminal scattering problems [4], which are wellknown benchmark models as well as proxies for realistic chemical reactions [42]. The results are compared against 


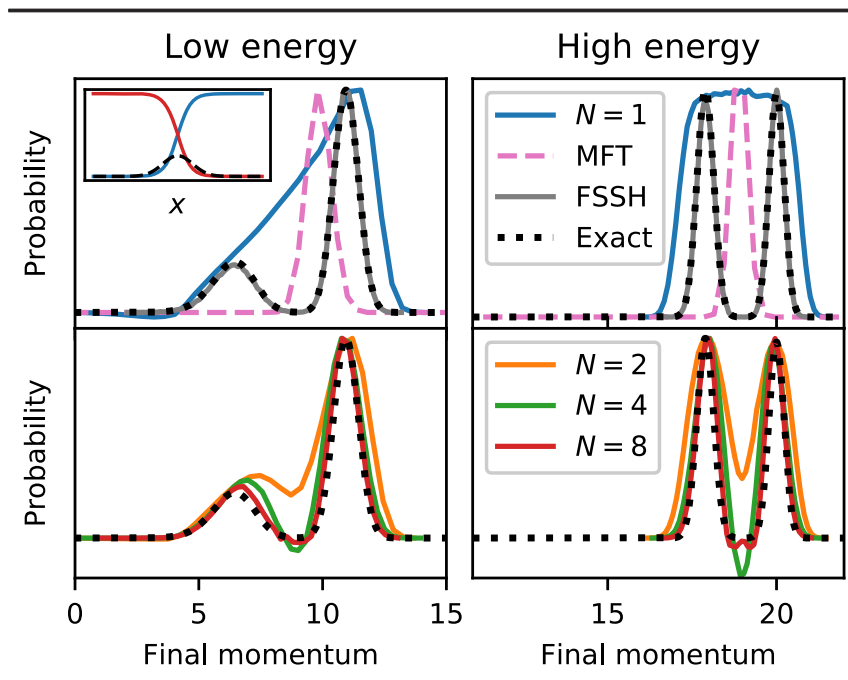

FIG. 2. Probability distribution of the nuclear momentum after an avoided crossing (model I). The inset shows the diabatic potentials (solid lines) and coupling (dashed line). A wave packet enters from the left on the lower surface with a narrow distribution of kinetic energies at roughly 1.5 (left panels) or 5 (right panels) times the asymptotic energy difference. Ehrenfest (MFT) gives a single peak around the average momentum, while linearized spin mapping $(N=1)$ envelopes the exact wavepacket distribution. For higher $N$, the spin path-integral method correctly reproduces the wave-packet branching.

calculations using numerically exact quantum mechanics as well as Ehrenfest dynamics and surface hopping. Simulation details are included in the Supplemental Material [41].

First, we consider the single avoided crossing (model I) in the form used by Miller and co-workers [43], with diabatic surfaces and coupling shown in the inset of Fig. 2. An initial Gaussian wave packet enters from the left on the lower surface, $\psi_{1}(x, t=0) \otimes|1\rangle$, with enough kinetic energy for both product channels to be open. Because of nonadiabatic coupling, the wave packet splits into two separate wave packets on the two surfaces and emerges as an entangled state, $\psi_{1}(x, t=\infty) \otimes|1\rangle+\psi_{2}(x, t=\infty) \otimes|2\rangle$, on the right. In Fig. 2 we show the distribution of the final momentum for two different initial energies. As is well known, Ehrenfest dynamics (MFT) is unable to capture the branching of the wave packet, whereas surface hopping (FSSH) provides a reasonably accurate description for this model. The $N=1$ simulation predicts an envelope that covers the full range of momenta allowed by energy conservation, but lacks the two-peak structure. However, by increasing $N$, we find that the distribution smoothly splits into two parts and thus recovers the correct entangled state.

We emphasize that the dynamics consists of independent and deterministic trajectories on a weighted average of the two states, similar to both Ehrenfest dynamics and the linearized spin-mapping method, and the key difference lies in the weighting of the trajectories. Because the weights may be positive or negative, some of these cancel out in

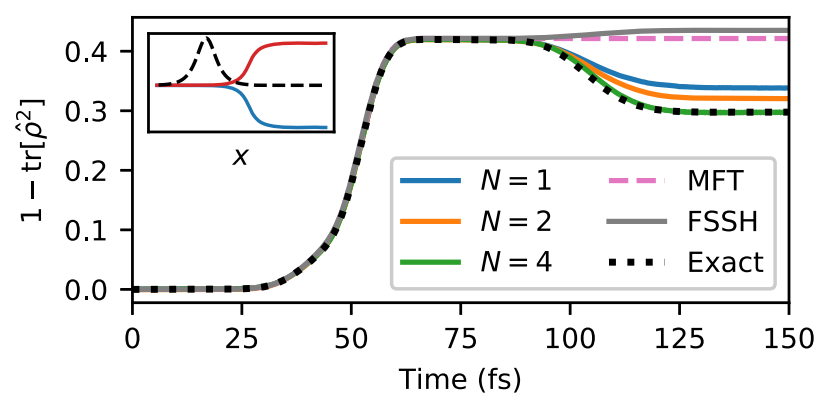

FIG. 3. Impurity in the extended coupling model (model III). The inset shows the adiabatic surfaces (solid) and nonadiabatic coupling (dashed). A wave packet enters from the left and is partly reflected. Only the spin path-integral method with $N=4$ is able to correctly describe the second crossing of the interaction region (results for $N=8$, not shown, overlay with $N=4$ ).

such a way that the ensemble branches when it emerges on uncoupled surfaces. This cancellation is reminiscent of more involved semiclassical methods such as Miller's forward-backward propagator, which is also known to capture wave-packet splitting in the present model [2224]. However, these approaches are inherently semiclassical, not classical, and include nuclear-coherence effects (to some level of approximation) via phases and prefactors that depend sensitively on the trajectory histories and make sampling difficult. The results of the simpler spin pathintegral method demonstrate that nuclear coherence is not necessary to recover the correct result. Although the trajectories also carry a sign, this depends in a relatively simple manner on a single degree of freedom, is fixed by the initial sampling, and is preserved by the dynamics.

For Tully's dual avoided crossing (model II) we reach the same conclusions, and in the Supplemental Material we show that the scattering probabilities are in good agreement with exact wave-packet calculations for a wide range of initial momenta [41].

Next, consider the more challenging extended coupling model (model III) shown in the inset of Fig. 3. As before, an initial wave packet enters on the lower surface from the left but now the total energy is low enough for the upper channel to be closed on the right. During the collision, it thus splits into a transmitted part on the lower surface and a part on the upper surface that reflects and passes through the interaction region a second time. Surface hopping is well known to fail dramatically for systems with recrossing, because the electronic amplitudes picked up during the first crossing are inconsistent with the active surfaces [5]. This "overcoherence" problem arises because the assumption of a unique trajectory for each electronic density matrix is not valid [44] and is related to neglecting quantum entanglement. Ehrenfest and various linearized mapping approaches have also been unable to describe this model [45].

To quantify the overcoherence problems of quantumclassical simulations, we have calculated the time evolution 


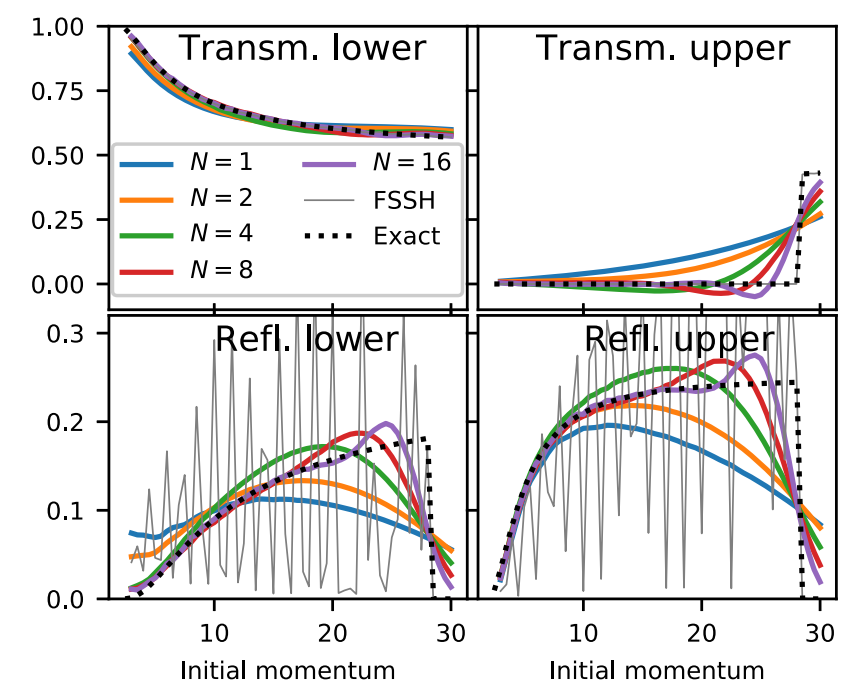

FIG. 4. Transmission and reflection probabilities onto the adiabatic states in the extended coupling model (model III). While surface hopping (FSSH) suffers from erroneous oscillations, the spin path-integral method appears to be converging smoothly with increasing $N$ towards the exact scattering probabilities from the log-derivative method [47].

of the impurity (linear entropy) $S_{L}=1-\operatorname{tr}\left[\hat{\rho}^{2}\right]=$ $2\left(\rho_{11} \rho_{22}-\left|\rho_{12}\right|^{2}\right)$, which is a measure of entanglement and is related to the decoherence indicator studied in Ref. [12] with coupled-trajectory simulations. Here, $\rho_{n m}$ denotes elements of the reduced density matrix in the adiabatic representation and the results are shown in Fig. 3. Ehrenfest completely misses the second crossing at about 100 fs (since its trajectories do not reflect), and although some surface-hopping trajectories do reflect, FSSH is unable to correctly describe the entanglement in this case. The spin path-integral method on the other hand reproduces the correct result.

Another well-known consequence of the overcoherence problems in surface hopping are erroneous oscillations [46] in the scattering probabilities as shown in Fig. 4. For the spin path-integral method, we observe that the calculated scattering probabilities converge towards the correct values with increasing $N$ (although reproducing the step as the upper channel opens appears to be difficult). Note that we did not need to add decoherence corrections for each trajectory (as is commonly done to fix surface hopping), but nevertheless do not observe the problems of overcoherence for the ensemble as a whole.

Finally, we note that unlike surface hopping, the results of the present method (like Ehrenfest and other mapping approaches $[22,48]$ ) are not dependent on whether the adiabatic or diabatic representation is used.

Conclusions. - In this Letter we have showed that features of quantum entanglement, such as wave-packet branching and impurity measurements, can indeed be captured by an ensemble of independent and deterministic classical trajectories. This discovery opens up a new class of mixed quantum-classical methods, as an alternative to surface-hopping, coupled-trajectory, or semiclassical simulations. It also extends the applicability of mapping approaches, which have been successful for predicting electronic coherences but so far have struggled to describe the nuclear dynamics of scattering problems. The presented method relies on positive and negative trajectory weights whose sign cancellation does not become more difficult for larger systems or longer simulation time. We therefore expect it to be applicable to complex molecular systems and condensed-phase problems.

Here we have limited the treatment to two-level systems, but a multilevel extension already exists for linearized spin mapping [21] and the spin path-integral extension is straightforward (although there is no guarantee that $G_{N}$ will depend on only a scalar variable). Since the SW formalism can be applied to any symmetry group [49], a similar treatment could be made also in systems with different symmetries.

Particularly interesting is the case where $\hat{\rho}$ is a thermal density matrix. Since the weights are preserved by the dynamics, we expect this to be useful for equilibrium dynamics as the quantum Boltzmann distribution will automatically be conserved. The details are left to a forthcoming paper.

The authors acknowledge support from the Swiss National Science Foundation through the NCCR MUST Network and from the Hans. H. Günthard scholarship. We also thank Annina Lieberherr, Joseph Lawrence, Jonathan Mannouch, and Graziano Amati for fruitful discussions.

*johan.runeson@phys.chem.ethz.ch jeremy.richardson@phys.chem.ethz.ch

[1] G. Stock and M. Thoss, Classical description of nonadiabatic quantum dynamics, Adv. Chem. Phys. 131, 243 (2005).

[2] Multitrajectory Ehrenfest is technically only a local meanfield theory because each trajectory feels a different effective potential.

[3] J. C. Tully, Perspective: Nonadiabatic dynamics theory, J. Chem. Phys. 137, 22A301 (2012).

[4] J. C. Tully, Molecular dynamics with electronic transitions, J. Chem. Phys. 93, 1061 (1990).

[5] J. E. Subotnik, A. Jain, B. Landry, A. Petit, W. Ouyang, and N. Bellonzi, Understanding the surface hopping view of electronic transitions and decoherence, Annu. Rev. Phys. Chem. 67, 387 (2016).

[6] C. F. Craig, W. R. Duncan, and O. V. Prezhdo, Trajectory Surface Hopping in the Time-Dependent Kohn-Sham Approach for Electron-Nuclear Dynamics, Phys. Rev. Lett. 95, 163001 (2005).

[7] J.E. Subotnik and N. Shenvi, A new approach to decoherence and momentum rescaling in the surface hopping algorithm, J. Chem. Phys. 134, 024105 (2011). 
[8] L. Wang, A. Akimov, and O. V. Prezhdo, Recent progress in surface hopping: 2011-2015, J. Phys. Chem. Lett. 7, 2100 (2016); G. Granucci and M. Persico, Critical appraisal of the fewest switches algorithm for surface hopping, J. Chem. Phys. 126, 134114 (2007).

[9] D. V. Shalashilin, Multiconfigurational Ehrenfest approach to quantum coherent dynamics in large molecular systems, Faraday Discuss. 153, 105 (2011).

[10] C. C. Martens, Surface hopping without momentum jumps: A quantum-trajectory-based approach to nonadiabatic dynamics, J. Phys. Chem. A 123, 1110 (2019).

[11] A. Abedi, N. T. Maitra, and E. K. U. Gross, Exact Factorization of the Time-Dependent Electron-Nuclear Wave Function, Phys. Rev. Lett. 105, 123002 (2010).

[12] S. K. Min, F. Agostini, and E. K. U. Gross, Coupledtrajectory Quantum-Classical Approach to Electronic Decoherence in Nonadiabatic Processes, Phys. Rev. Lett. 115, 073001 (2015).

[13] F. Agostini, S. K. Min, A. Abedi, and E. K. U. Gross, Quantum-classical nonadiabatic dynamics: Coupled- vs independent-trajectory methods, J. Chem. Theory Comput. 12, 2127 (2016).

[14] B. F. E. Curchod and T. J. Martínez, Ab initio nonadiabatic quantum molecular dynamics, Chem. Rev. 118, 3305 (2018).

[15] A. Kelly, R. van Zon, J. Schofield, and R. Kapral, Mapping quantum-classical Liouville equation: Projectors and trajectories, J. Chem. Phys. 136, 084101 (2012).

[16] O. V. Prezhdo and C. Brooksby, Quantum Backreaction Through the Bohmian Particle, Phys. Rev. Lett. 86, 3215 (2001); B. F. Curchod and I. Tavernelli, On trajectory-based nonadiabatic dynamics: Bohmian dynamics versus trajectory surface hopping, J. Chem. Phys. 138, 184112 (2013); D. D. Holm, J. I. Rawlinson, and C. Tronci, The Bohmion method in nonadiabatic quantum hydrodynamics, J. Phys. A 54, 495201 (2021).

[17] W. H. Miller and C. W. McCurdy, Classical trajectory model for electronically nonadiabatic collision phenomena. A classical analog for electronic degrees of freedom, J. Chem. Phys. 69, 5163 (1978).

[18] H.-D. Meyer and W.H. Miller, A classical analog for electronic degrees of freedom in nonadiabatic collision processes, J. Chem. Phys. 70, 3214 (1979).

[19] G. Stock and M. Thoss, Semiclassical Description of Nonadiabatic Quantum Dynamics, Phys. Rev. Lett. 78, 578 (1997).

[20] J. E. Runeson and J. O. Richardson, Spin-mapping approach for nonadiabatic molecular dynamics, J. Chem. Phys. 151, 044119 (2019).

[21] J.E. Runeson and J. O. Richardson, Generalized spin mapping for quantum-classical dynamics, J. Chem. Phys. 152, 084110 (2020).

[22] X. Sun and W. H. Miller, Semiclassical initial value representation for electronically nonadiabatic molecular dynamics, J. Chem. Phys. 106, 6346 (1997).

[23] W. H. Miller, Electronically nonadiabatic dynamics via semiclassical initial value methods, J. Phys. Chem. A 113, 1405 (2009).

[24] W. H. Miller, Perspective: Quantum or classical coherence? J. Chem. Phys. 136, 210901 (2012).
[25] N. Makri, Forward-backward semiclassical and quantum trajectory methods for time correlation functions, Phys. Chem. Chem. Phys. 13, 14442 (2011).

[26] P. J. Ollitrault, G. Mazzola, and I. Tavernelli, Nonadiabatic Molecular Quantum Dynamics with Quantum Computers, Phys. Rev. Lett. 125, 260511 (2020).

[27] C. Brif and A. Mann, Phase-space formulation of quantum mechanics and quantum-state reconstruction for physical systems with Lie-group symmetries, Phys. Rev. A 59, 971 (1999).

[28] A. B. Klimov and S. M. Chumakov, A Group-Theoretical Approach to Quantum Optics: Models of Atom-Field Interactions (Wiley-VCH, Weinheim, 2009).

[29] R. L. Stratonovich, On distributions in representation space, Sov. Phys. JETP 4, 891 (1957), http://jetp.ras.ru/cgi-bin/e/ index/e/4/6/p891? a=list.

[30] H.-D. Meyer and W. H. Miller, Classical models for electronic degrees of freedom: Derivation via spin analogy and application to $\mathrm{F}^{*}+\mathrm{H}_{2} \rightarrow \mathrm{F}+\mathrm{H}_{2}$, J. Chem. Phys. 71, 2156 (1979).

[31] J. R. Mannouch and J. O. Richardson, A partially linearized spin-mapping approach for nonadiabatic dynamics. I. Derivation of the theory, J. Chem. Phys. 153, 194109 (2020); A partially linearized spin-mapping approach for nonadiabatic dynamics. II. Analysis and comparison with related approaches, J. Chem. Phys. 153, 194110 (2020).

[32] U. Müller and G. Stock, Consistent treatment of quantummechanical and classical degrees of freedom in mixed quantum-classical simulations, J. Chem. Phys. 108, 7516 (1998).

[33] S. J. Cotton and W. H. Miller, Symmetrical windowing for quantum states in quasi-classical trajectory simulations: Application to electronically non-adiabatic processes, J. Chem. Phys. 139, 234112 (2013).

[34] T. P. Fay, L. P. Lindoy, D. E. Manolopoulos, and P. J. Hore, How quantum is radical pair magnetoreception? Faraday Discuss. 221, 77 (2020).

[35] O. Bramley, C. Symonds, and D. V. Shalashilin, Quantum system-bath dynamics with quantum superposition sampling and coupled generalized coherent states, J. Chem. Phys. 151, 064103 (2019); X. He and J. Liu, A new perspective for nonadiabatic dynamics with phase space mapping models, J. Chem. Phys. 151, 024105 (2019).

[36] L.S. Schulman, Techniques and Applications of Path Integration (Wiley, New York, 1981).

[37] A. Altland and B. D. Simons, Condensed Matter Field Theory (Cambridge University Press, Cambridge, England, 2010).

[38] J. H. Wilson and V. Galitski, Breakdown of the Coherent State Path Integral: Two Simple Examples, Phys. Rev. Lett. 106, 110401 (2011).

[39] H. Kleinert, Path Integrals in Quantum Mechanics, Statistics, Polymer Physics and Financial Markets, 5th ed. (World Scientific, Singapore, 2009).

[40] Let $\hat{U}$ be a unitary representation of the rotation $s \rightarrow s^{\prime}$, then $\hat{w}\left(\boldsymbol{s}^{\prime}\right)=\hat{U} \hat{w}(\boldsymbol{s}) \hat{U}^{-1}$ and the invariance follows from the cyclicity of the trace.

[41] See Supplemental Material at http://link.aps.org/supplemental/ 10.1103/PhysRevLett.127.250403 for simulation details and additional results. 
[42] L. M. Ibele and B. F. E. Curchod, A molecular perspective on Tully models for nonadiabatic dynamics, Phys. Chem. Chem. Phys. 22, 15183 (2020).

[43] N. Ananth, C. Venkataraman, and W. H. Miller, Semiclassical description of electronically nonadiabatic dynamics via the initial value representation, J. Chem. Phys. 127, 084114 (2007).

[44] J. E. Subotnik, W. Ouyang, and B. R. Landry, Can we derive Tully's surface-hopping algorithm from the semiclassical quantum Liouville equation? Almost, but only with decoherence, J. Chem. Phys. 139, 214107 (2013).

[45] X. Gao, M. A. C. Saller, Y. Liu, A. Kelly, J. O. Richardson, and E. Geva, Benchmarking quasiclassical mapping Hamiltonian methods for simulating electronically nonadiabatic molecular dynamics, J. Chem. Theory Comput. 16, 2883 (2020).
[46] J. E. Subotnik and N. Shenvi, Decoherence and surface hopping: When can averaging over initial conditions help capture the effects of wave packet separation? J. Chem. Phys. 134, 244114 (2011).

[47] D. E. Manolopoulos, An improved log derivative method for inelastic scattering, J. Chem. Phys. 85, 6425 (1986); M. H. Alexander, G. Parlant, and T. H. Hemmer, A log-derivative propagation scheme for the exact solution of two-state curve crossing problems, J. Chem. Phys. 91, 2388 (1989).

[48] S. J. Cotton, R. Liang, and W. H. Miller, On the adiabatic representation of Meyer-Miller electronic-nuclear dynamics, J. Chem. Phys. 147, 064112 (2017).

[49] T. Tilma, M. J. Everitt, J. H. Samson, W. J. Munro, and K. Nemoto, Wigner Functions for Arbitrary Quantum Systems, Phys. Rev. Lett. 117, 180401 (2016). 